\title{
Feasibility of a Hydraulic Power Assist System for Use in Hybrid Neuroprostheses
}

\author{
Kevin M. Foglyano, ${ }^{1}$ Rudi Kobetic, ${ }^{1}$ Curtis S. To, ${ }^{2}$ \\ Thomas C. Bulea, ${ }^{2}$ John R. Schnellenberger, ${ }^{1}$ Musa L. Audu, ${ }^{2}$ \\ Mark J. Nandor, ${ }^{1}$ Roger D. Quinn, ${ }^{3}$ and Ronald J. Triolo ${ }^{1,2,4}$ \\ ${ }^{1}$ Advanced Platform Technology Center, Louis Stokes Cleveland Department of Veterans Affairs Medical Center, \\ Cleveland, OH 44106, USA \\ ${ }^{2}$ Department of Biomedical Engineering, Case Western Reserve University, Cleveland, OH 44106, USA \\ ${ }^{3}$ Department of Mechanical and Aerospace Engineering, Case Western Reserve University, Cleveland, OH 44106, USA \\ ${ }^{4}$ Department of Orthopaedics, Case Western Reserve University, Cleveland, OH 44106, USA
}

Correspondence should be addressed to Kevin M. Foglyano; kfoglyano@fescenter.org

Received 5 June 2014; Revised 3 February 2015; Accepted 1 March 2015

Academic Editor: Amir A. Zadpoor

Copyright ( 2015 Kevin M. Foglyano et al. This is an open access article distributed under the Creative Commons Attribution License, which permits unrestricted use, distribution, and reproduction in any medium, provided the original work is properly cited.

Feasibility of using pressurized hydraulic fluid as a source of on-demand assistive power for hybrid neuroprosthesis combining exoskeleton with functional neuromuscular stimulation was explored. Hydraulic systems were selected as an alternative to electric motors for their high torque/mass ratio and ability to be located proximally on the exoskeleton and distribute power distally to assist in moving the joints. The power assist system (PAS) was designed and constructed using off-the-shelf components to test the feasibility of using high pressure fluid from an accumulator to provide assistive torque to an exoskeletal hip joint. The PAS was able to provide $21 \mathrm{Nm}$ of assistive torque at an input pressure of $3171 \mathrm{kPa}$ with a response time of $93 \mathrm{~ms}$ resulting in $32^{\circ}$ of hip flexion in an able-bodied test. The torque output was independent of initial position of the joint and was linearly related to pressure. Thus, accumulator pressure can be specified to provide assistive torque as needed in exoskeletal devices for walking or stair climbing beyond those possible either volitionally or with electrical stimulation alone.

\section{Introduction}

Restoring walking is one of the main desires of individuals who have a spinal cord injury (SCI) [1]. Thus, there is a significant effort in the field of orthotics to restore locomotion, including walking on uneven terrain and negotiating stairs. There are a number of approaches to restoring upright mobility after paralysis from SCI including mechanical bracing [2], functional neuromuscular stimulation (FNS) [3-5], powered exoskeletons [6-9], and hybrid systems $[10,11]$ that combine two or more of these approaches.

Recently, there has been a surge in powered exoskeleton development, including commercial devices such as Rewalk and Ekso [12,13]. Exoskeletons are able to drive the motion of the limbs for walking and stair climbing with external motors.
However, they are limited by the amount of power and the weight of the torque generating motors that must be carried. In addition, they do not provide the physiological benefits of exercise from actively contracting the otherwise paralyzed muscles.

Therefore, there has been an increased effort in hybrid system development that combines either surface [14] or implanted FNS [15] with exoskeleton's on-demand external power to provide physiological benefits and to minimize external power requirements. There are a number of ways to provide external power including the use of electric motors $[12,13]$, pneumatic [16-18] and elastic elements [19-21] to supply joint torques. Other approaches store energy from stimulated knee extensors and transfer it to drive hip motion using springs and pneumatic components [22, 23]. 
In this study, feasibility of a power assist system (PAS) using a hydraulic circuit with pressurized fluid to provide assistive torque for joint movement was designed and tested. We hypothesized that this PAS could be a viable alternative in providing assistive torque for joint movements.

\section{Materials and Methods}

2.1. Power Assist System Design. As a test bed for bench testing, the PAS (Figure 1) was incorporated into the right hip mechanism of the existing exoskeleton [15] (Figure 2). The PAS utilizes a single pump to pressurize fluid in an accumulator and a directional control valve to distribute it to the hydraulic pistons located at the joints. The previously designed variable constraint hip mechanism [24] utilizes a hydraulic circuit to unlock the swing hip by opening appropriate valves while the stance hip is locked to provide postural support. In this design iteration, linear displacement of the hydraulic cylinder, which was attached to the thigh upright, by the pressurized fluid generates torque at the right hip joint by means of a rack and pinion transmission.

While the PAS system can be applied to any hydraulic circuit, its operation in this design configuration (Figure 2) is as follows. Two valves ( $\mathrm{H} 1$ and $\mathrm{H} 2$ ) allow the ability to lock, unlock, and isolate the right hip cylinder (RHC) used for torque generation from the PAS. The blind side of the RHC is connected to the pressurized accumulator (PA) by opening the valve P1 and shifting the position of a closed-center 4-way, 3-position solenoid actuated directional control valve (DCV) to the right. Flow from the PA pressurizes the blind side of the $\mathrm{RHC}$, resulting in RHC piston extension which produces a hip flexion torque through the rack and pinion transmission. Fluid from the rod side of the cylinder flows through the open valve $\mathrm{H} 2$ into the hip accumulator (HA).

Once the power assist torque is no longer required, the DCV is deenergized shifting the valve left to the closed center position, and the system returns to its default state. To recharge PA to prepare for the next cycle, the pump is activated to transfer fluid from the tank accumulator (TA) to PA. This pressurizes PA to its initial condition (measured by the pump side pressure transducer) and at the same time reduces the pressure of TA below its original system resting pressure. The fluid volume pumped from TA to PA is equal to that displaced from PA to the blind side of the RHC during the hip flexion assist. Next, the DCV is shifted in the opposite direction and valves $\mathrm{H} 1$ and P1 are opened to channel the flow into TA from HA. Since TA is below the system resting pressure and the rest of the circuit is above, the excess fluid from the previous PAS injection period stored in HA will then flow back into TA bringing the entire system to its initial condition and ready for the next cycle when needed.

2.2. Power Assist System Specifications. To specify individual components of the PAS, biomechanical requirements of stair ascent in normal individuals were examined while considering the limitations of weakened muscles. A minimum of $60^{\circ}$ hip flexion angle is necessary for a person of an average stature to clear a standard stair step riser height of $20 \mathrm{~cm} \mathrm{[26].}$ Approximately $23 \mathrm{Nm}$ of hip flexion moment is necessary

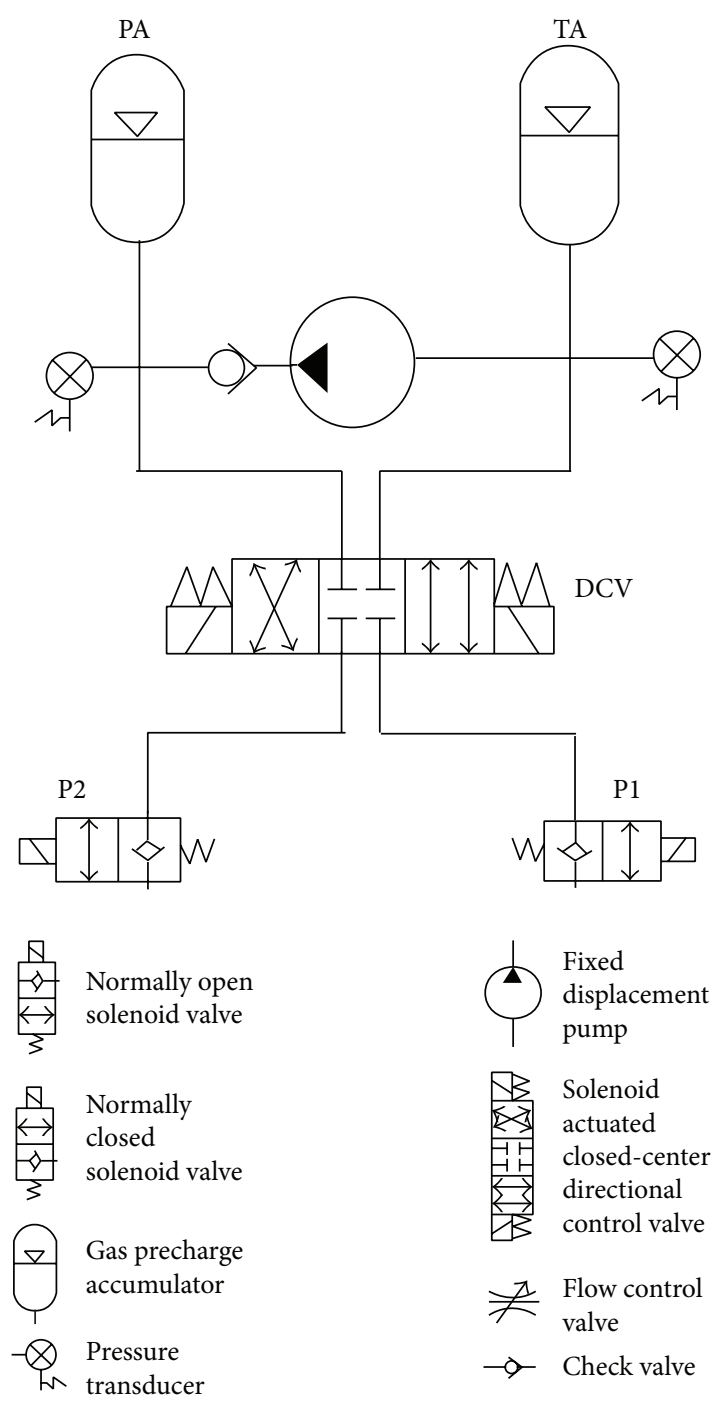

FIgURE 1: Diagram of the PAS system. Note that P1 and P2 can connect to any hydraulic circuit. In this study, the PAS is connected to a single hydraulic actuator via valve P1.

to hold a leg at $60^{\circ}$ hip flexion for a $79 \mathrm{~kg}$ individual [26]. Therefore, we specified PAS components to generate $25 \mathrm{Nm}$ of torque at $60^{\circ}$ of hip flexion. The volume $\left(V_{\mathrm{PA}}\right)$ of the hydropneumatic accumulator, PA, derived from Boyle's law, can be expressed as in (1) (note that the influences of temperature and system efficiency were not factored into the derivation of (1) and the compressible fluid in the accumulator is nitrogen). Consider the following:

$$
V_{\mathrm{PA}}=\frac{A_{\text {blind }} r \Delta \theta_{\text {hip }}}{0.9^{1 / n}-\left(0.9\left(\tau_{\text {end }}+p_{\text {rest }} A_{\text {rod }} r\right) / p_{\text {ic }} A_{\text {blind }} r\right)^{1 / n}},
$$

where $A_{\text {blind }}$ is the RHC piston blind area, $A_{\text {rod }}$ is the RHC piston rod area, $r$ is linear-to-rotary rack and pinion transmission ratio $(0.55 \mathrm{~mm} / \mathrm{deg}[15]), \Delta \theta_{\text {hip }}$ is the estimated hip angle excursion that may require power assist, $n$ is the polytropic index (1.4 for nitrogen assuming an adiabatic process), $\tau_{\text {end }}$ is the output torque at the end of the hip 
motion $(25 \mathrm{Nm}), p_{\text {rest }}$ is the system resting pressure ( $\left.414 \mathrm{kPa}\right)$, and $p_{\text {ic }}$ is the initial pressure of PA and corresponds to the operating pressure rating of the pump. Thus, the objective is to minimize $p_{\text {ic }}$ so that the power requirements of the pump are minimized. Equation (1) can be solved for $p_{\text {ic }}$, to get

$$
p_{\text {ic }}=\frac{0.9\left(\tau_{\text {end }}+p_{\text {rest }} A_{\text {rod }} r\right)}{A_{\text {blind }} r\left(0.9^{1 / n}-A_{\text {blind }} r \Delta \theta_{\text {hip }} / V_{\mathrm{PA}}\right)^{n}} .
$$

All the variables on the right side of (2) are known except for $V_{\mathrm{PA}}$ and $\Delta \theta_{\text {hip }}$. Knowing this and assuming the values are as stated above, it can be seen upon inspection of (2) that $p_{\text {ic }}$ decreases with an increasing $V_{\mathrm{PA}}$ and decreases with a decreasing $\Delta \theta_{\text {hip }}$. Thus, the goal is to minimize the required $\Delta \theta_{\text {hip }}$ and choose a $V_{\mathrm{PA}}$ such that it is small enough to be portable but large enough so that $p_{\text {ic }}$ is kept low. For example, if we assume that power assist is necessary from 0 to $60^{\circ}$ hip flexion and choose a small diaphragm accumulator of $0.075 \mathrm{~L}$ (Hydac, Bethlehem, PA) for PA, $p_{\text {ic }}$ would need to be approximately $3213 \mathrm{kPa}$. In this case, when the DCV and $\mathrm{P} 1$ are opened to connect PA to the cylinder at $0^{\circ}$ hip flexion, $3213 \mathrm{kPa}$ of fluid pressure at the cylinder produces $35 \mathrm{Nm}$ of hip flexion torque by means of the linear-to-rotary transmission. The pressure drops to $2413 \mathrm{kPa}$ at $60^{\circ}$ hip flexion to produce $25 \mathrm{Nm}$.

Another critical specification of the pump is the displacement or flow output. A high displacement allows for a faster recharge time of PA. In this example, if we assume that it is necessary to recharge PA within one second, the pump displacement needed is $12.9 \mathrm{~cm}^{3} / \mathrm{s}$ at $2413 \mathrm{kPa}$. However, if we assume power assist is only necessary from 30 to $60^{\circ}$ hip flexion and choose a larger accumulator of $0.16 \mathrm{~L}, p_{\text {ic }}$ would drop to $2565 \mathrm{kPa}$. In this case, at the onset of power assist, $2565 \mathrm{kPa}$ at the cylinder produces $27 \mathrm{Nm}$ of hip flexion torque at $30^{\circ}$ hip flexion. The pressure then decreases to $2413 \mathrm{kPa}$ at $60^{\circ}$ hip flexion to produce $25 \mathrm{Nm}$. Since $\Delta \theta_{\text {hip }}$ is reduced to half of that in the previous example, the required pump displacement is also reduced by half to $6.45 \mathrm{~cm}^{3} / \mathrm{s}$ at $2413 \mathrm{kPa}$.

A major advantage of a hydraulic power source is the potential weight reduction. Hydraulic systems have been shown to have torque/mass ratios many times greater than that of electric motors and other power sources [27]. In addition, the system described here contains just one motor/pump unit that can be placed proximally on the exoskeleton and direct power distally to the joints as needed as opposed to having a motor at each joint. By locating the pump proximally the need for additional assistive power due to the inertial demands of distally located weight is minimized. Keeping the mass proximal allows it to be directly transferred to the ground and is not carried by the user or an external power source [25]. Suitable wearable miniature hydraulic pumps are currently being developed [28] and adding this unit to our current exoskeleton prototype will keep the weight (Table 1) at or below clinically available electrically driven exoskeletons [29].

2.3. Power Assist System Simulation. A computer model was created in MATLAB (The MathWorks, Inc., Natick, MA, USA) using SimMechanics, SimHydraulics, and SimScape
TABLE 1: Weight of HNP + PAS*

\begin{tabular}{lc}
\hline Structure & $6.5 \mathrm{~kg}$ \\
Hydraulics & $7.4 \mathrm{~kg}$ \\
Battery/electronics & $1.5 \mathrm{~kg}$ \\
Pump ${ }^{*}[28]$ & $2.75 \mathrm{~kg}$ \\
\hline Total & $18.15 \mathrm{~kg}$ \\
\hline
\end{tabular}

Weight of entire hybrid neuroprosthesis with * ${ }^{*}$ pump for power assist system added.

Toolboxes of Simulink to verify the proposed PAS design. In the simulation, the PAS was connected to an anatomically realistic model of the human leg which simulated hip flexion moment on movement produced through either volitional contraction or FNS. A hip flexion torque of $20 \mathrm{Nm}$ was applied to the leg model 1 second after initiating the simulation which resulted in a hip rotation of $25^{\circ}$. The simulated hydraulic valves of the PAS model were then opened to expose the cylinder to the high pressure accumulator to apply an external assistive hip flexion torque, which resulted in a further increase in hip flexion to the specified angle of $60^{\circ}$ (Figure 3).

2.4. Power Assist System Bench Testing. A prototype of the hydraulic PAS was constructed from off-the-shelf components for bench testing. For simplicity, only the right hip was included for bench testing as shown schematically in Figure 2. The directional control valve (DCV) (Hyvair Corporation, Magnolia, TX) and solenoid valves P1 and H1 (Allenair Corporation, Mineola, NY) controlled fluid flow between pressurized accumulator PA and the $2.2 \mathrm{~cm}$ bore double-acting hydraulic cylinder RHC (Clippard Minimatic, Cincinnati, OH). The power of pressurized fluid stored in PA, a $0.075 \mathrm{~L}$ diaphragm style accumulator (Hydac, Bethlehem, PA), was released to produce force on the cylinder's piston which provided torque at the hip by means of a rack and pinion transmission.

Torque, angle, and angular velocity measurements were made with a Biodex System 3 robotic dynamometer (Biodex Medical Systems, Shirley, NY). Pressure sensors (Gems Sensors \& Controls, Plainville, CT) were attached to monitor pressure of accumulators PA and TA at the blind and rod side of the cylinder. Data were collected with custom circuitry integrated into the MATLAB XPC target prototyping environment which executes Simulink models in real time. A NI 6071E DAQ card (National Instruments, Austin, TX) inside of the target PC acquired torque, angle, and angular velocity from the Biodex. In addition, the DAQ card delivered digital output signals from the $\mathrm{xPC}$ target to control the valves.

The hydraulic cylinder attached to the thigh upright provided hip flexion by linear displacement of the piston through the rack and pinion transmission. The thigh upright of the brace was attached to the Biodex arm (Figure 2). The entire hydraulic system with all valves in the open position was precharged to $414 \mathrm{kPa}$ to minimize the compliance from any residual air bubbles left in the system. The high pressure accumulator (PA) was pressurized with a hand pump with 


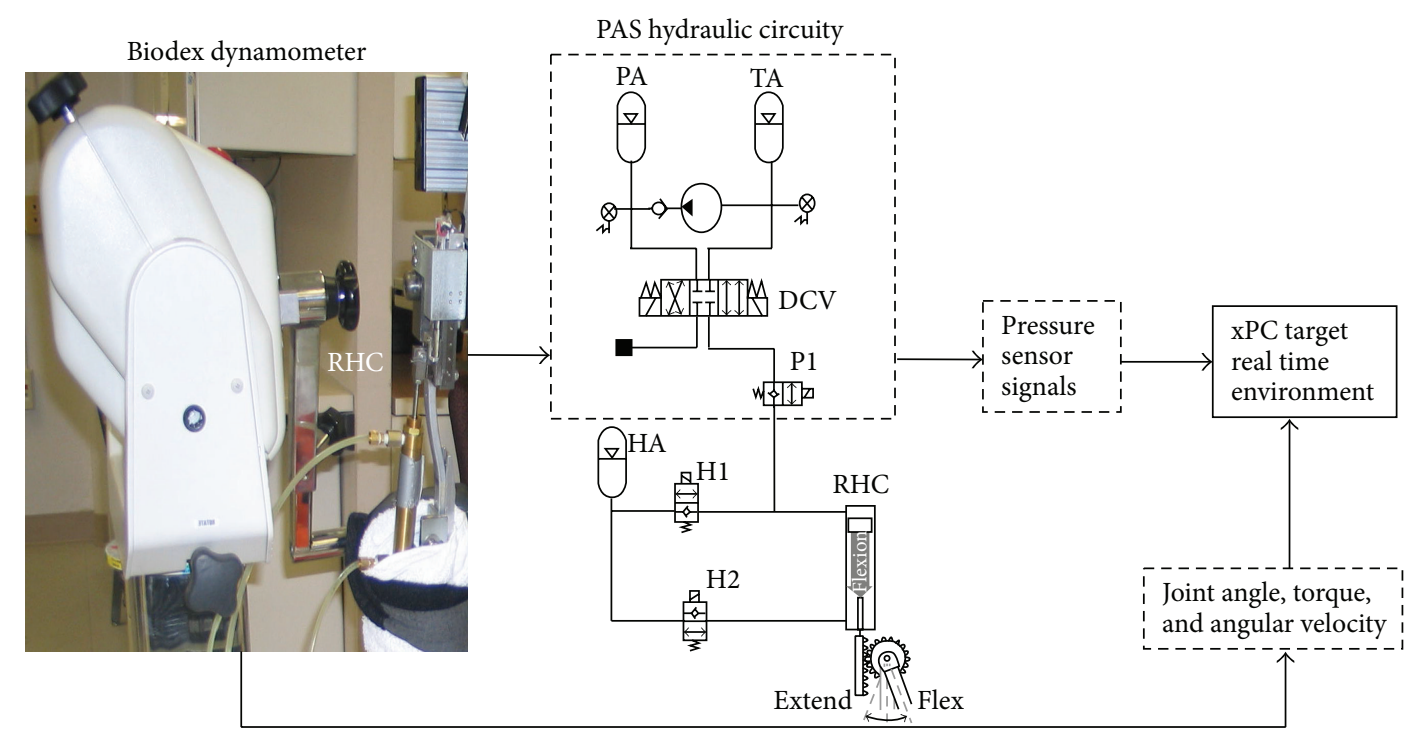

FIGURE 2: PAS bench testing setup. The PAS hydraulic circuitry is connected to the rotary actuator consisting of right hip cylinder (RHC) connected between the exoskeleton upright and rack and pinion transmission to transmit torque. The upright is attached to a Biodex dynamometer to measure flexion torque. Pressure, torque, angle, and angular velocity data are read into an xPC Simulink real time environment.

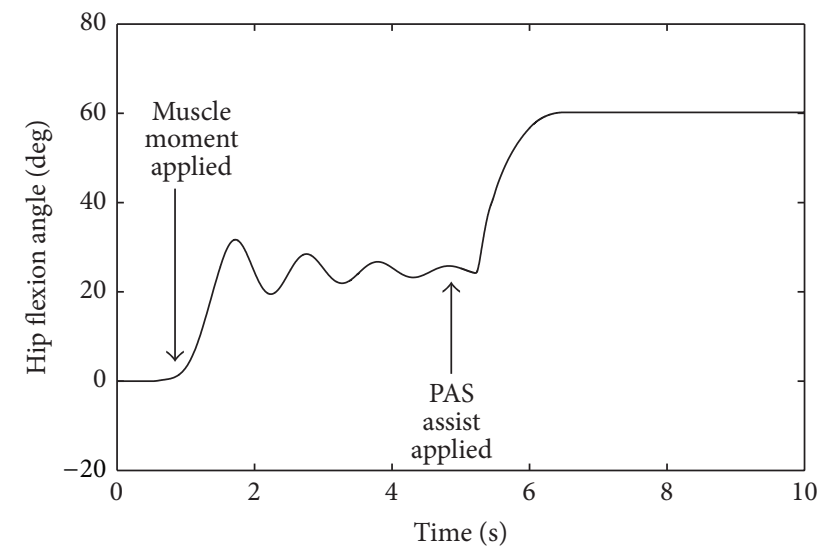

FIGURE 3: Simulated PAS operation. Hip angle of $25^{\circ}$ was achieved before activation and assistive power of PAS was simulated to drive hip to $60^{\circ}$ of flexion.

all valves in their default states as seen in Figure 2. Once the desired pressure was reached, the DCV and appropriate hydraulic valves were opened to expose the right hip cylinder to the high pressure which applied torque at the hip. The DCV was shifted to the right and P1 was opened for fluid flow from the PA to the blind side of the RHC to extend the cylinder which flexed the hip. The excess fluid from the rod side of the cylinder went into the accumulator HA. In a portable design a motorized pump would move fluid from TA to PA. Once the flexion stage was complete, the DCV was shifted to the left while the $\mathrm{P} 1$ stayed open and $\mathrm{H} 2$ closed to shift the excess fluid back into TA. The PA was pressurized to $689,1379,2068,2758$, and $3172 \mathrm{kPa}$ (maximum safe rating for components used) for isometric tests at 0 and $60^{\circ}$ of hip flexion. In addition, isokinetic tests limited to $120^{\circ}$ / s were performed to measure the differential pressure in the cylinder to calculate the torque throughout the entire range of motion.

To determine passive resistance of the hydraulic circuit of the PAS, the hip was flexed through the range of motion by pulling vertically on a load cell through a rope which was attached to the brace upright for six trials. The force measured by the load cell was multiplied by the perpendicular distance from the line of force application to the hip center to calculate the moment required to flex the hip. This distance was found by attaching reflective markers at the hip center, the point of force application on the upright and on the rope using a Vicon (Oxford, UK) motion capture system to track the movement and determine the changing moment arm. This was repeated for six trials with the hydraulic cylinder detached from the rack and pinion transmission to find the difference in moment caused by the passive resistance of the hydraulic circuit.

2.5. Power Assist System Human Testing. To simulate PAS operation with an SCI subject, an able-bodied volunteer $(165 \mathrm{~cm}, 64 \mathrm{~kg})$ gave her informed consent and was fitted with the brace. Reflective markers were placed on the trunk corset and leg upright and the Vicon motion capture system was used to capture the marker trajectories at $120 \mathrm{~Hz}$ and body segments were created from the marker data to calculate joint angles. The high pressure accumulator was charged to $3172 \mathrm{kPa}$. To simulate voluntary muscle weakness or the actions of FNS, the subject was instructed to flex her right hip to approximately $45^{\circ}$ after which the PAS was activated to complete the movement while she was instructed to provide minimal resistance or assistance. Six trials with PAS were performed and the data were averaged across the six trials. 


\section{Results}

3.1. Power Assist System Bench Testing. The input pressure supplied to the PA accumulator and the output isometric hip flexion torque at 0 and $60^{\circ}$ is shown in Figure 4 . There is a linear relationship between the input pressure and the output torque and the output torque is practically independent of hip flexion angle. Therefore, we can control the applied hip flexion torque by controlling the pressure of the accumulator. Figure 5 shows a typical change in hip angle, torque, and cylinder pressure differential in the isokinetic tests. When a pressure of $3172 \mathrm{kPa}$ was released with the orthosis in full hip extension, the resulting torque of about $20 \mathrm{Nm}$ moved the hip into $50^{\circ}$ of hip flexion. The torque decreased as the hip moved into flexion due to the decrease in pressure with extension of the hydraulic cylinder flexing the hip.

The mean angular velocity of the hip was $77 \pm 8^{\circ} / \mathrm{s}$ with a mean flow rate of $16.5 \pm 1.6 \mathrm{~cm}^{3} / \mathrm{s}$. The average system response time was $93 \pm 23 \mathrm{~ms}$ from the activation of the DCV to onset of hip movement. Passive resistance of the PAS circuit was found to be less than $4 \mathrm{Nm}$ throughout the range of motion.

3.2. Power Assist System Human Testing. In the able-bodied experiments, the PAS increased hip flexion angle by a mean of $32 \pm 5^{\circ}$ across the six trials. Photographs before and after the PAS was activated are shown in Figure 6 to illustrate the additional hip flexion that was provided following volitional flexion.

\section{Discussion and Conclusions}

A hydraulic source of assistive power was designed and tested in this study. The PAS was able to provide $21 \mathrm{Nm}$ of torque to supplement hip flexion in a hybrid system combining weak voluntary muscle activity and an exoskeleton. More importantly, our results validate a linear relationship between input pressure and output torque that is independent of joint angle. For preliminary assessment, the accumulator was pressurized using a simple hand pump; however, automated pumps and motors capable of producing required pressures with reasonable weight and power consumption for portable use are available [28] and can be integrated into the PAS in the future now that feasibility of the approach has been established.

The torque values measured during bench testing were lower than the values calculated from the mathematical equations and model $(21 \mathrm{Nm}$ as compared to the predicted $25 \mathrm{Nm}$ at $60^{\circ}$ ). This difference is likely due to the passive resistance caused by friction in the hydraulic system and the linear-to-rotary transmission. The pressure drop across the cylinder was higher than predicted from the model (ending pressure of approximately $1379 \mathrm{kPa}$ as compared to the predicted $2413 \mathrm{kPa}$ ). This is likely due to residual air in the system and suboptimal component selection. Custom fittings or a manifold designed specifically for the circuit has the potential to reduce the number of hydraulic connectors and the amount of tubing which could minimize the pressure losses and reduce passive resistance.

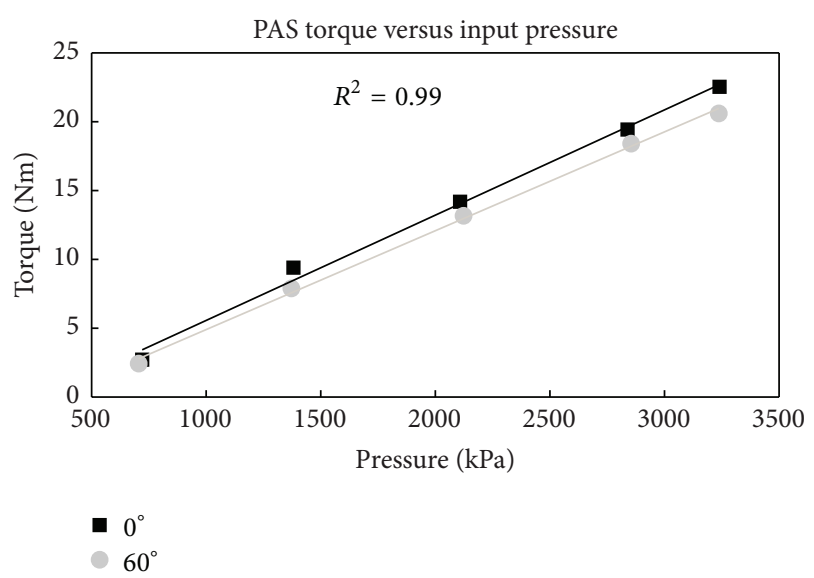

FIGURE 4: Output isometric torque as a function of supplied pressure at 0 and $60^{\circ}$ of hip flexion. $R^{2}$ of 0.99 is representative of both curves.

We were able to achieve $32^{\circ}$ of assistive hip flexion in the able-bodied testing. While it would have been beneficial to have electromyogram (EMG) recordings of the subject to ensure there was no volitional assist once the PAS was activated, the major muscles responsible for hip flexion are deep within the pelvis and not easily accessible for EMG recording. Thus, the able-bodied testing was only to approximate the results for an SCI subject using FNS and continued clinical testing with volunteers with paraplegia is necessary.

From the results of the bench testing, we can extrapolate and predict pressures that would be required to produce a desired hip flexion moment. Knowing the relationship between input pressure and torque and the pressure drop across the range of motion, the pressure required to achieve desired hip flexion motion can be specified. For example, if it takes $23 \mathrm{Nm}$ of hip flexion moment at $60^{\circ}$ for a leg to clear a standard step [26] plus additional $15 \mathrm{Nm}$ for the $5 \mathrm{~kg}$ knee-ankle-foot orthosis [15], then an input pressure of approximately $6440 \mathrm{kPa}$ would be required to flex the hip from $0^{\circ}$ to $60^{\circ}$ assuming the worst case scenario of no volitional or stimulated input from the user. This includes the pressure of $5240 \mathrm{kPa}$ required to generate $38 \mathrm{Nm}$ extrapolated from Figure 4, in addition to compensating for the observed pressure drop of $20 \mathrm{kPa} /{ }^{\circ}$ as the hip flexes (Figure 5). However, if a stimulated response can achieve $25 \mathrm{Nm}$ at $60^{\circ}$ [26], the remaining torque could be added by using an input pressure of approximately $3447 \mathrm{kPa}$ to supplement the stimulation. All of these pressures are achievable and safe with proper component selection; however, this feasibility testing was limited to the existing setup and components and was constrained to a maximum of $3172 \mathrm{kPa}$ due to the limitations of the accumulators and tubing of our current hydraulic system.

Preliminary results show that using readily available offthe-shelf hydraulic components it is feasible to integrate a power assist system into a hybrid neuroprosthesis in order to provide additional hip flexion torque to assist in lifting and supporting the braced leg to a desired height. Controllers 


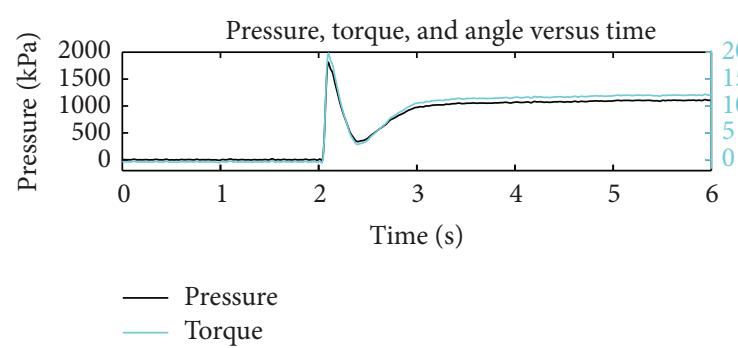

(a)

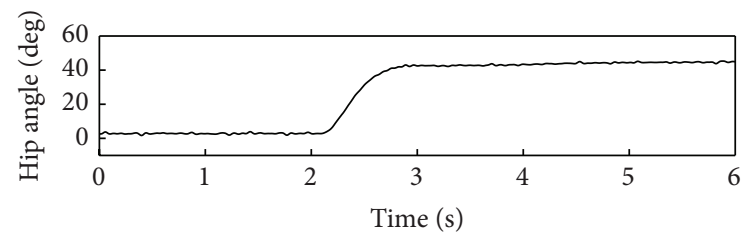

(b)

FIGURE 5: Cylinder pressure differential and torque change (a) during isokinetic (120\%) movement of the hip (b). Power assist was activated at time $=2$ seconds.

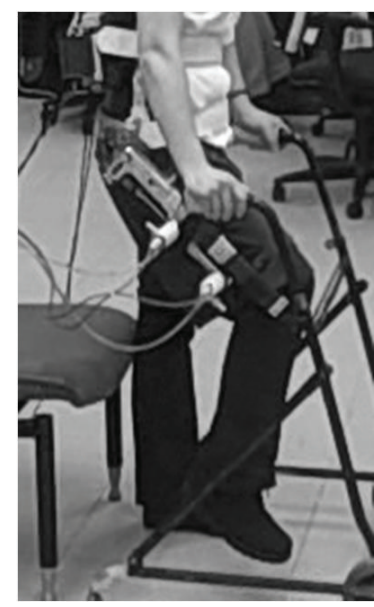

(a)

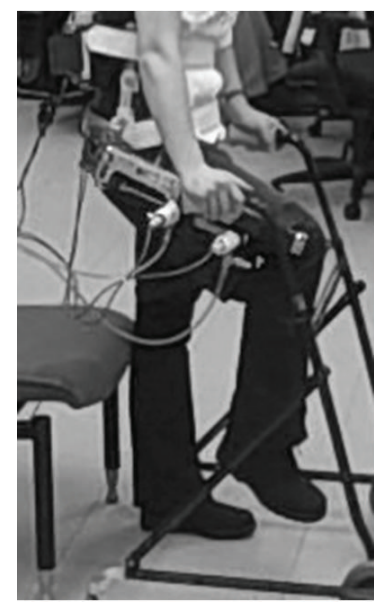

(b)

FIGURE 6: Able-bodied PAS test. Subject simulated FNS by flexing hip to approximately $45^{\circ}$ (a). PAS was then activated to continue flexing the hip to approximately $77^{\circ}$ with minimal user assistance or resistance (b).

can be developed to combine power assist on demand with muscle stimulation [30] to maximize physiological benefits of stimulation and minimize external power requirements.

An advantage of this hydraulic design is that, with proper valve configuration [24], it is possible to transmit hip flexion power from one hip to another to assist with contralateral extension and to the knee through hip-knee coupling without requiring a dedicated motor at each joint. Thus, a similar system that contains just one motor/pump unit can be constructed to direct power to whichever joint it needed as opposed to using a motor at each joint to be actuated.

There are three major advantages of using flow discharged from an accumulator as opposed to using flow directly generated from a pump. First, pressure does not have to build from a low pressure which would result in a slow application of torque. High pressure is applied to the cylinder immediately after the DCV has opened which will enable a response time comparable to stimulated muscle. While the average system response time from valve activation to limb movement was $93 \mathrm{~ms}$, isometric testing found that the time from valve activation to $90 \%$ of the maximum torque output was $159 \pm 45 \mathrm{~ms}$, which falls within range of stimulated muscle response times $(100-300 \mathrm{~ms})$ [31]. This response time was independent of input pressure but likely will be influenced by component selection (e.g., tubing and valve switching speed) and any compliance or residual air bubbles in the system. Due to this quick response time, a small fixed displacement pump can be used rather than a larger and costlier pressure-compensated variable displacement pump. The second advantage of using an accumulator to discharge the flow is that pump activation can be kept separate from valve activation. This reduces the maximum instantaneous electric power requirements to drive the hydraulic system. And third one is for safety in that the hydraulic exoskeleton worn by the user is never directly coupled to the pump which will reduce the risk of a malfunctioning pump injecting too much pressure which could harm the individual by moving the joints to undesired positions.

Since the PAS operation is feasible, future studies need to concentrate on quantifying system performance in volunteers with SCI in conjunction with FNS. The PAS approach can also be applied without stimulation to assist physiological or household ambulators with partial paralysis or significant muscle weakness and limited voluntary movement. Future technical development should include system optimization to minimize the weight and power consumption of the system 
and maximize the pressure and displacement that the pump supplies to accumulator.

\section{Abbreviations}

DCV: Directional control valve

EMG: Electromyogram

FNS: Functional neuromuscular stimulation

HNP: Hybrid neuroprosthesis

PA: Pressurized accumulator

PAS: Power assist system

RHC: Right hip cylinder

SCI: Spinal cord injury

TA: Tank accumulator.

\section{Conflict of Interests}

The authors have declared that no competing interests exist.

\section{Acknowledgments}

This material was based on work supported in part by the Department of Veterans Affairs, Rehabilitation Research and Development Service (Grant nos. A6404R and B0608R), and the Department of Defense, Congressionally Directed Medical Research Program (Grant no. PR043074).

\section{References}

[1] D. L. Brown-Triolo, M. J. Roach, K. Nelson, and R. J. Triolo, "Consumer perspectives on mobility: implications for neuroprosthesis design," Journal of Rehabilitation Research and Development, vol. 39, no. 6, pp. 659-669, 2002.

[2] A. V. Nene, H. J. Hermens, and G. Zilvold, "Paraplegic locomotion: a review," Spinal Cord, vol. 34, no. 9, pp. 507-524, 1996.

[3] M. R. Popovic, A. Curt, T. Keller, and V. Dietz, "Functional electrical stimulation for grasping and walking: indications and limitations," Spinal Cord, vol. 39, no. 8, pp. 403-412, 2001.

[4] A. Kralj, T. Bajd, R. Turk, J. Krajnik, and H. Benko, "Gait restoration in paraplegic patients: a feasibility demonstration using multichannel surface electrode FES," Journal of Rehabilitation Research \& Development, vol. 20, no. 1, pp. 3-20, 1983.

[5] R. Kobetic and E. B. Marsolais, "Synthesis of paraplegic gait with multichannel functional neuromuscular stimulation," IEEE Transactions on Rehabilitation Engineering, vol. 2, no. 2, pp. 6679, 1994.

[6] H. A. Quintero, R. J. Farris, C. Hartigan, I. Clesson, and M. Goldfarb, "A powered lower limb orthosis for providing legged mobility in paraplegic individuals," Topics in Spinal Cord Injury Rehabilitation, vol. 17, no. 1, pp. 25-33, 2011.

[7] G. Zeilig, H. Weingarden, M. Zwecker, I. Dudkiewicz, A. Bloch, and A. Esquenazi, "Safety and tolerance of the ReWalk exoskeleton suit for ambulation by people with complete spinal cord injury: a Pilot Study," Journal of Spinal Cord Medicine, vol. 35, no. 2, pp. 96-101, 2012.

[8] P. D. Neuhaus, J. H. Noorden, T. J. Craig, T. Torres, J. Kirschbaum, and J. E. Pratt, "Design and evaluation of Mina: a robotic orthosis for paraplegics," in Proceedings of the IEEE International Conference on Rehabilitation Robotics (ICORR '11), pp. 1-8, Zurich, Switzerland, July 2011.
[9] L. Mertz, “The next generation of exoskeletons: lighter, cheaper devices are in the works," IEEE Pulse, vol. 3, no. 4, pp. 56-61, 2012.

[10] A. J. del-Ama, A. D. Koutsou, J. C. Moreno, A. de-los-Reyes, Á. Gil-Agudo, and J. L. Pons, "Review of hybrid exoskeletons to restore gait following spinal cord injury," Journal of Rehabilitation Research and Development, vol. 49, no. 4, pp. 497-514, 2012.

[11] J. C. Moreno, A. J. del Ama, A. de los Reyes-Guzmán, Á. GilAgudo, R. Ceres, and J. L. Pons, "Neurorobotic and hybrid management of lower limb motor disorders: a review," Medical \& Biological Engineering \& Computing, vol. 49, no. 10, pp. 11191130, 2011.

[12] A. Esquenazi, M. Talaty, A. Packel, and M. Saulino, "The Rewalk powered exoskeleton to restore ambulatory function to individuals with thoracic-level motor-complete spinal cord injury," The American Journal of Physical Medicine and Rehabilitation, vol. 91, no. 11, pp. 911-921, 2012.

[13] S. A. Kolakowsky-Hayner, J. Crew, S. Moran, and A. Shah, "Safety and feasibility of using the ekso bionic exoskeleton to aid ambulation after spinal cord injury," Spine, vol. 4, pp. 3-11, 2013.

[14] K. H. Ha, H. A. Quintero, R. J. Farris, and M. Goldfarb, "Enhancing stance phase propulsion during level walking by combining fes with a powered exoskeleton for persons with paraplegia," in Proceedings of the 34th Annual International Conference of the IEEE Engineering in Medicine and Biology Society (EMBS '12), pp. 344-347, September 2012.

[15] R. Kobetic, C. S. To, J. R. Schnellenberger et al., "Development of hybrid orthosis for standing, walking, and stair climbing after spinal cord injury," Journal of Rehabilitation Research and Development, vol. 46, no. 3, pp. 447-462, 2009.

[16] B. G. do Nascimento, C. B. S. Vimieiro, D. A. P. Nagem, and M. Pinotti, "Hip orthosis powered by pneumatic artificial muscle: voluntary activation in absence of myoelectrical signal," Artificial Organs, vol. 32, no. 4, pp. 317-322, 2008.

[17] S. Kang, J. Ryu, G. Kim, and M. Mun, "Hip joint control of PGO for paraplegics," Key Engineering Materials, vol. 326-328, pp. 735-738, 2006.

[18] C. L. Lewis and D. P. Ferris, "Invariant hip moment pattern while walking with a robotic hip exoskeleton," Journal of Biomechanics, vol. 44, no. 5, pp. 789-793, 2011.

[19] S. Carda, M. Invernizzi, G. Cognolato, E. Piccoli, A. Baricich, and C. Cisari, "Efficacy of a hip flexion assist orthosis in adults with hemiparesis after stroke," Physical Therapy, vol. 92, no. 5, pp. 734-739, 2012.

[20] S. Charooni, M. O. Tokhi, and B. Heller, "The use of elastic element in a hybrid orthosis for swing phase generation in orthotic gait," in Proceedings of the 5th Annual Conference of the International Functional Electrical Simulation Society, Aalborg, Denmark, June 2000.

[21] J. S. Sulzer, R. A. Roiz, M. A. Peshkin, and J. L. Patton, "A highly backdrivable, lightweight knee actuator for investigating gait in stroke," IEEE Transactions on Robotics, vol. 25, no. 3, pp. 539548, 2009.

[22] A. Kangude, B. Burgstahler, J. Kakastys, and W. Durfee, "Single channel hybrid FES gait system using an energy storing orthosis: preliminary design," in Proceedings of the 31st Annual International IEEE EMBS Conference, 2009.

[23] W. K. Durfee and A. Rivard, "Design and simulation of a pneumatic, stored-energy hybrid orthosis for gait restoration," Journal of Biomechanical Engineering, vol. 127, no. 6, pp. 10141019, 2005. 
[24] C. S. To, R. Kobetic, J. R. Schnellenberger, M. L. Audu, and R. J. Triolo, "Design of a variable constraint hip mechanism for a hybrid neuroprosthesis to restore gait after spinal cord injury," IEEE/ASME Transactions on Mechatronics, vol.13, no. 2, pp. 197205, 2008.

[25] H. Kazerooni, "Exoskeletons for human power augmentation," in Proceedings from the IEEE International Conference on Intelligent Robots and Systems (IROS '05), pp. 3120-3125, August 2005.

[26] R. Kobetic, E. B. Marsolais, and P. C. Miller, "Function and strength of electrically stimulated hip flexor muscles in paraplegia," IEEE Transactions on Rehabilitation Engineering, vol. 2, no. 1, pp. 11-17, 1994.

[27] J. M. Hollerbach, I. W. Hunter, and J. Ballantyne, "A comparative analysis of actuator technologies for robotics," in Robotics Review 2, O. Khatib, J. J. Craig, and T. Lozano-Pérez, Eds., pp. 299-342, MIT Press, Cambridge, Mass, USA, 1992.

[28] W. Durfee, J. Xia, and E. Hsiao-Wecksler, "Tiny hydraulics for powered orthotics," in Proceedings of the 12th Annual IEEE International Conference of Rehabilitation Robotics, July 2011.

[29] M. Bortole, Design and control of a robotic exoskeleton for gait rehabilitation [M.S. thesis], The Charles III University of Madrid, Madrid, Spain, 2013.

[30] A. J. del-Ama, J. C. Moreno, Á. Gil-Agudo, A. de-los-Reyes, and J. L. Pons, "Online assessment of human-robot interaction for hybrid control of walking," Sensors, vol. 12, no. 1, pp. 215-225, 2012.

[31] R. Kobetic, E. B. Marsolais, P. Samame, and G. Borges, "The next step: artificial walking," in Human Walking, J. Rose and J. G. Gamble, Eds., pp. 225-252, Williams \& Wilkins, Baltimore, Md, USA, 1994. 

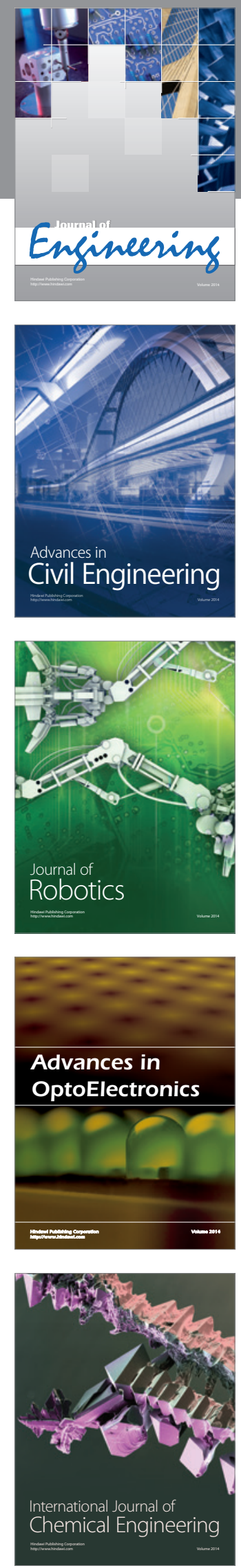

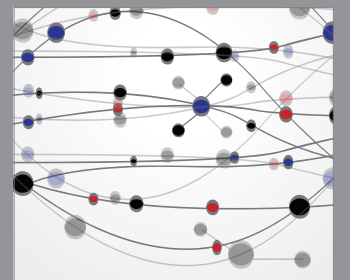

The Scientific World Journal
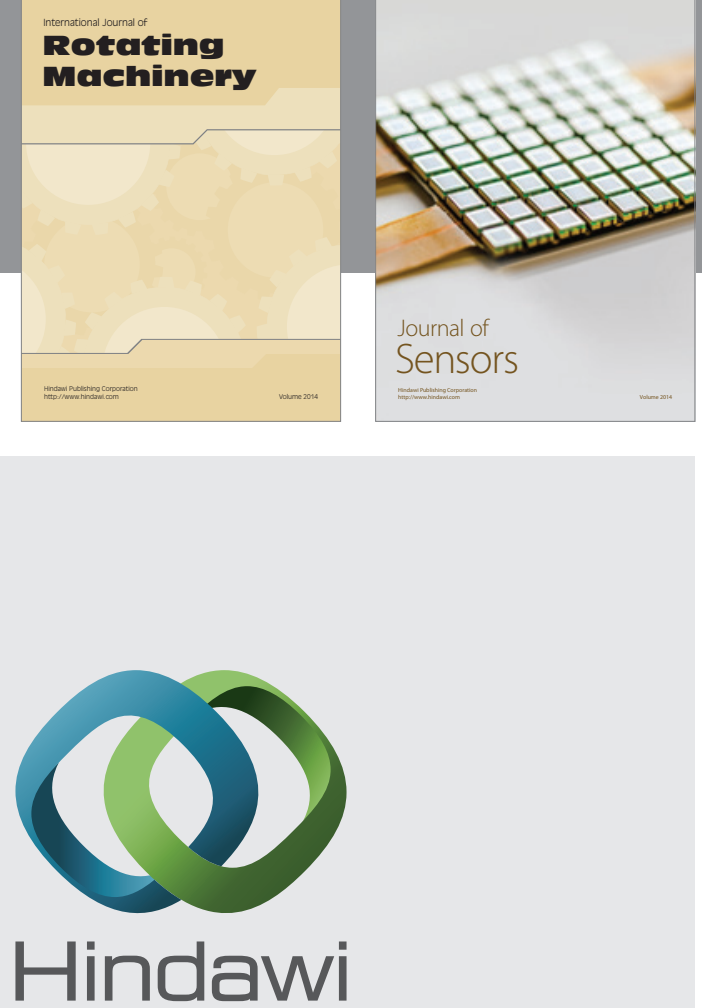

Submit your manuscripts at http://www.hindawi.com
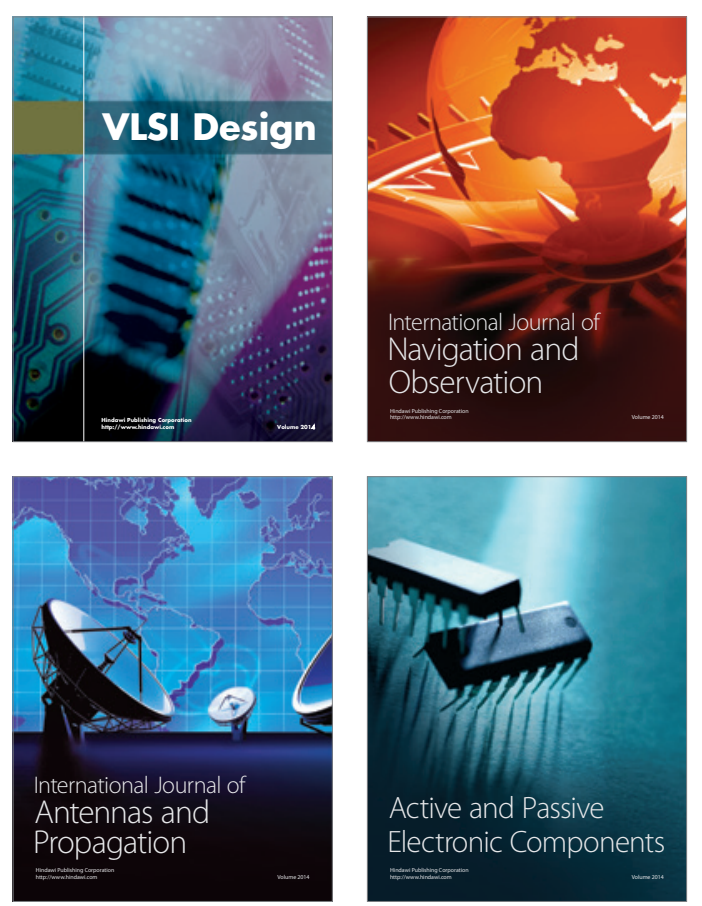
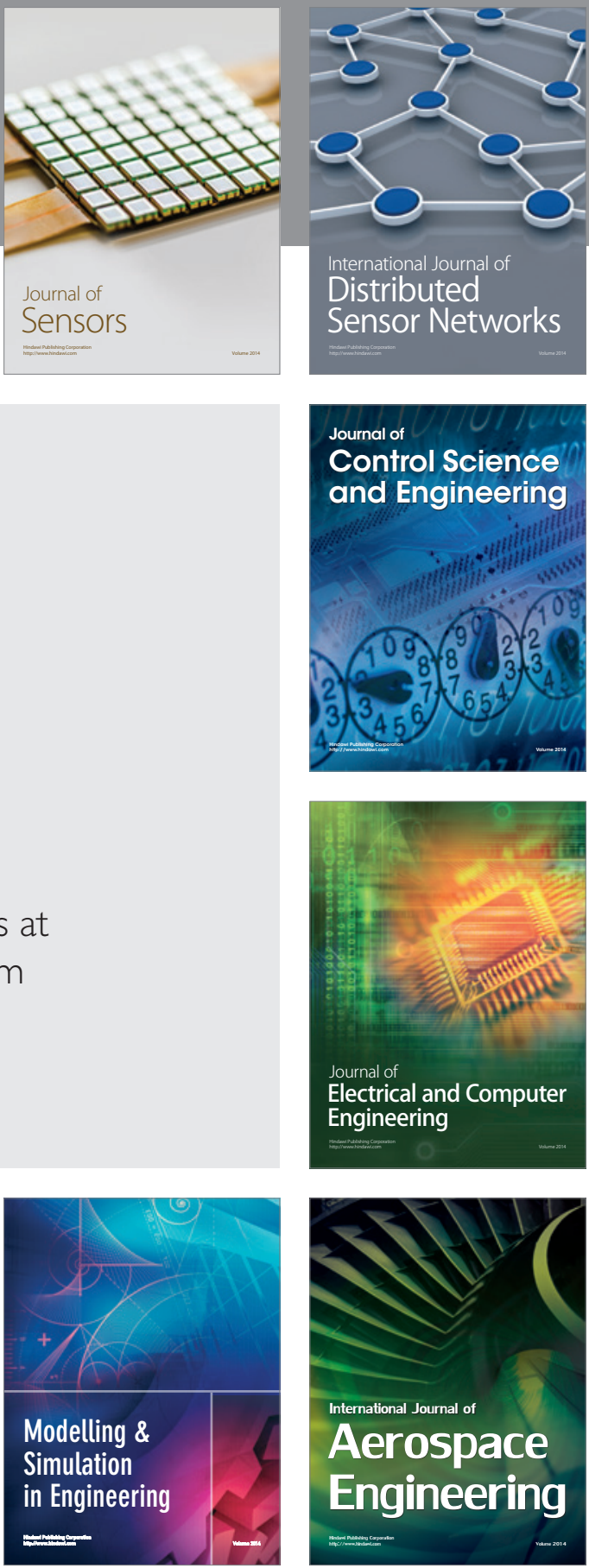

Journal of

Control Science

and Engineering
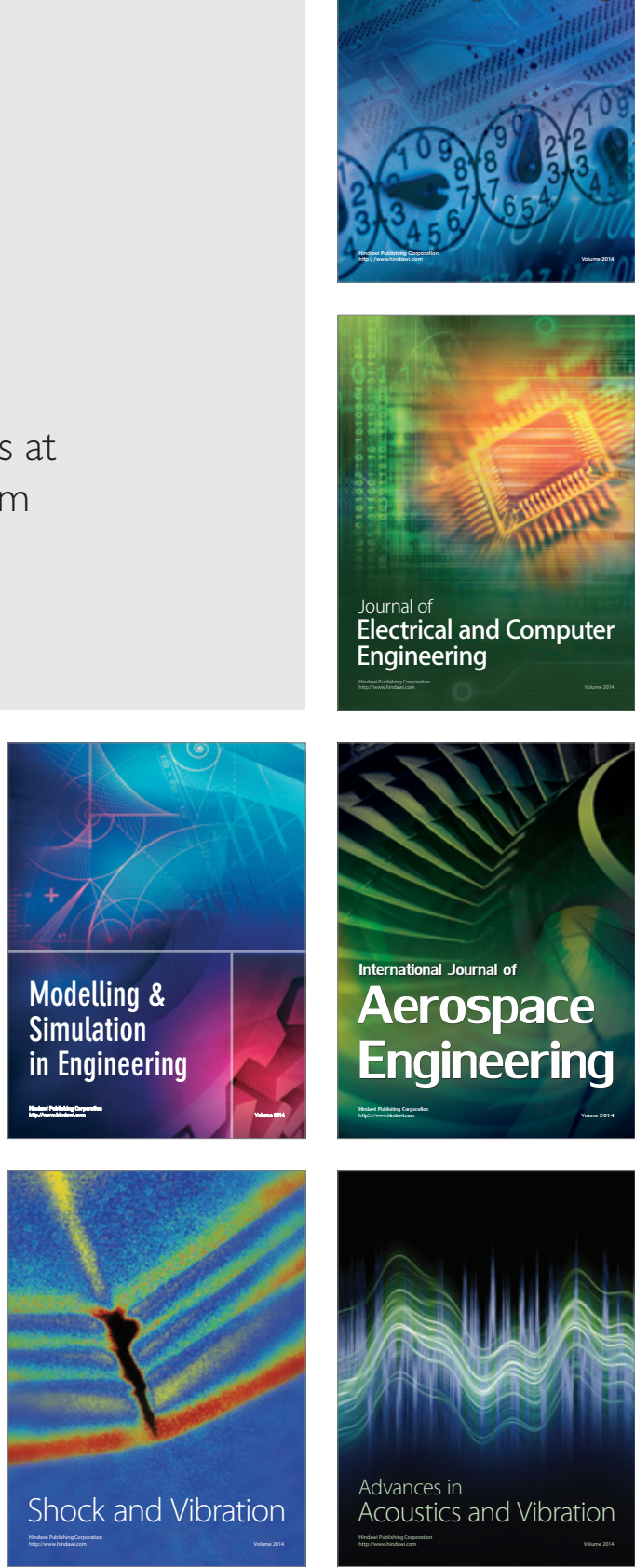